\title{
Density Functional Study of Electronic and Structural Properties of Gold-Cadmium Selenide/Telluride Nanoclusters
}

\author{
Shimeles T. Bulbula ${ }^{1,2}$ and Hagos W. Zeweldi ${ }^{3}$ \\ ${ }^{1}$ School of Material Science and Engineering, Wuhan University of Technology, No. 205 Luoshi Road, Wuhan, Hubei 430070, China \\ ${ }^{2}$ Department of Physics, Woldia University, Woldia, Ethiopia \\ ${ }^{3}$ Department of Physics, Mekelle University, Mekelle, Ethiopia
}

Correspondence should be addressed to Shimeles T. Bulbula; shime7250@gmail.com

Received 26 February 2015; Revised 27 April 2015; Accepted 27 April 2015

Academic Editor: Aziz Dinia

Copyright (C) 2015 S. T. Bulbula and H. W. Zeweldi. This is an open access article distributed under the Creative Commons Attribution License, which permits unrestricted use, distribution, and reproduction in any medium, provided the original work is properly cited.

\begin{abstract}
Semiconductor nanowires are one class of building blocks that show promise for application in nanoscale electronics. Metalsemiconductor nanowire helps to improve the electrical properties or create unique ones. Electronic and structural properties of cadmium selenide/telluride connected to gold electrode clusters have been the focus of this research due to their importance in constructing fast microelectric devices. The simulations were carried out by using VASP (Vienna Ab-Initio Simulation Package) which utilizes the method of density functional theory (DFT) and plane wave basis set. Optimization was performed to obtain the minimum energy structure. In this research paper the result shows that the HOMO-LUMO gaps for the minimum energy cadmium selenide/telluride connected to gold electrodes decrease as cluster size increases, whereas the binding energy shows a reverse relationship with the cluster size. However, a few clusters show special properties like $\mathrm{AuCd}_{2} \mathrm{Se}_{3}$ and $\mathrm{AuCd}_{2} \mathrm{Te}_{3}$ clusters.
\end{abstract}

\section{Introduction}

Nanotechnology is the design, characterization, production, and application of materials, devices, and systems by controlling shape and size at the nanoscale. The prefix nanoin the word nanotechnology means a billionth $\left(1.0 \times 10^{-9}\right)$ [1]. Richard Feynman in 1959 at Caltech gave a lecture entitled "There's plenty of room at the bottom" [2] that provided the vision of exciting new discoveries if one could fabricate materials or devices at the atomic or molecular size. The science got started in early 1980s with two major developments, that is, the birth of cluster science and the invention of the Scanning Tunneling Microscope (STM) [3].

Any particle somewhere between 3 and $3.0 \times 10^{7}$ atoms is considered as cluster. Clusters are a special class of matter in which the limited size leads to unusual combinations of physical and chemical properties. For instance, it has been found that the materials that are neither magnetic nor superconducting in bulk phase could be magnetic or superconducting in the nanophase [4]. When a bulk metal is reduced in size to a few hundred atoms, the density of states in the conduction band containing electrons changes dramatically resulting in the continuous density of states in the band being replaced by a set of discrete energy levels which may have energy level spacing larger than the thermal energy $k_{B} T$ [5]. The special properties of nanoparticles are due quantum size confinement in nanostructure and extremely large surface to volume ratio (area/volume $>1$ ) relative to bulk materials. This means a greater proportion of atoms are found at the surface compared to those inside. This will be helpful for strong chemical bonding is present, delocalization of valence electrons can be extensive, and the extent of delocalization can vary with the size the system.

The two basic approaches in making nanostructure involve either a top-down approach whereby an existing solid is gradually reduced in size using some external radiation like UV radiation and/or chemical and a bottom-up approach whereby the nanostructure is built atom by atom, molecule by molecule, or cluster by cluster. However, bottom-up approach is a better chance to obtain nanostructures with lower defect, more homogeneous chemical composition, and better short 
and long range ordering [6]. Therefore, in this paper the bottom-up approach is used to make the clusters.

Semiconductor nanomaterials are one of the most active areas of today. Many of their properties are very dependent on size, for example, optical transitions can be tuned simply by changing the size of the clusters [7]. Selenium (Se) and tellurium (Te) are elementary semiconductors. CdSe and CdTe are compound (II-VI) semiconductors which are used in computer chips, optoelectronics devices, transistors, and also medical imaging. The selective growth of gold tips onto the apexes of CdSe semiconductor nanorods provides enhancement or new functionality to the nanostructure [8]. In this work, Vienna Ab-Initio Simulation Package (VASP) is used as the simulation tool for electronic structures of the cadmium selenide ( $\mathrm{CdSe}$ ) and cadmium telluride $(\mathrm{CdTe})$ clusters connected to gold electrodes.

\section{Computational Details}

The simulations were carried out by using VASP [9] (Vienna $A b$-Initio Simulation Package). The combination of DFT, plane wave basis set [10], and pseudopotentials [11] has become a well-established methodology in electronic structure calculation. Therefore, we used the first-principles density functional theory calculations [12] for electronic structure and total energy within the Local Density Approximation (LDA) self-consistent method [13] for the exchange correlation functional [14]. The wave functions are expanded in plane waves with an energy cutoff taken from the default maximum value in the POTCAR [9] file (PREC = high) $(\sim 200-400 \mathrm{eV})$. The simple cubic cell is of $20 \AA$ length with a periodic boundary condition. The clusters were positioned at the center of the cell, and the $\Gamma$ point approximation for Brillouin zone [15] sampling and also structural optimizations (minimizing the Kohn-Sham energy functional) were performed using a conjugate gradient method [16]. The simulation process in this work contains the steps: First; we have been constructed the cluster in a super cell. Then, geometry optimization has been done; finally, we have been obtained density of state (DOS) and energy of the cluster.

\section{Results and Discussion}

\subsection{Electronic Structure Calculation}

3.1.1. Electronic Structure of Dimers. The binding energy is the amount of energy released at its creation or the amount of energy you should need to add to the system in order to break it up. The binding energy per pair, BE, of a cluster $\left(A_{n} B_{n}\right)$ can be calculated as [17]

$$
\mathrm{BE}=\frac{\left(n E(A)+n E(B)-E\left(A_{n} B_{n}\right)\right)}{n} ;
$$

for a cluster $A_{n} B_{m} C_{r}$, the binding energy per atom is calculated by

$$
\mathrm{BE}=\frac{\left(n E(A)+m E(B)+r E(C)-E\left(A_{n} B_{m} C_{r}\right)\right)}{(n+m+r)},
$$

where $n, r$, and $m$ are integers $1,2,3, \ldots$.
TABLE 1: Bond length, binding energy, and HOMO-LUMO gap of dimers.

\begin{tabular}{lccc}
\hline Dimer & BL $(\AA)$ & BE $(\mathrm{eV})$ & $\begin{array}{c}\text { HOMO-LUMO } \\
\text { gaps }(\mathrm{eV})\end{array}$ \\
\hline $\mathrm{Au}_{2}$ & 2.47 & 3.12 & 1.95 \\
$\mathrm{Te}_{2}$ & 2.54 & 4.80 & 2.64 \\
$\mathrm{Se}_{2}$ & 2.16 & 5.75 & 3.27 \\
$\mathrm{Cd}_{2}$ & 3.09 & 2.22 & 3.15 \\
$\mathrm{CdSe}$ & 2.32 & 2.29 & 0.31 \\
$\mathrm{AuCd}$ & 2.55 & 1.46 & 2.81 \\
$\mathrm{AuSe}$ & 2.26 & 3.99 & 1.57 \\
$\mathrm{CdTe}$ & 2.50 & 1.90 & 0.27 \\
\hline
\end{tabular}

The energy difference between the HOMO and LUMO is termed as the bandgap, calculated by

$$
\operatorname{HOMO-LUMOgap}\left(E_{g}\right)=E_{\mathrm{LUMO}}-E_{\mathrm{HOMO}}
$$

Table 1 describes the numerical values of bond length, binding energy and HOMO-LUMO gap of dimers studied in this work. The bond length of a dimer depends on the atomic radius of each atom. As we know the atomic radii become smaller as you move across a row or period but larger as you move down the period; for instance, Se and Te are found in the same group but in periods IV and V, respectively, since $r_{a}(\mathrm{Se})<r_{a}(\mathrm{Te})$ results bond length of CdSe dimer is smaller than CdTe dimer.

The bond length of Se-Se, Te-Te, and Au-Au are found to be in good agreement with experimental values of $2.17 \AA$, $2.56 \AA$, and $2.47 \AA$ [18], respectively [19]. The binding energy and the bond length of CdSe are found similar to the values calculated by Sellers et al. [20] and Sanville et al. [21]. The bond length of CdTe is found approach result to the value calculated by Bhattacharya and Kshirsagar [22].

Generally, Table 1 helps to analyze the properties of the dimers of the elementary particles which form the clusters studied in this work.

\subsubsection{Electronic Calculation of CdSe and CdTe Dimers Coupled} with Au Atom. In this research paper the triangular geometry structures of AuCdSe and AuCdTe are preferable due to being more stable than the linear ones.

The addition of the Au atom to the CdSe dimer changes the electronic structure of CdSe (Figure 1(a)) including bond length. The bond length of CdSe, in the presence of the $\mathrm{Au}$ atom, is found increasing from $2.32 \AA$ to $2.58 \AA$ with $\mathrm{Au}-\mathrm{Cd}$ and Au-Se bond lengths equal to $2.58 \AA$ and $2.34 \AA$, respectively. Similarly, the addition of the $\mathrm{Au}$ atom to the CdTe dimer (Figure 1(b)); results indicated that changing the electronic structure of the CdTe dimer, even the CdTe bond length is changed. The bond length of CdTe in the presence of the Au atom is found increasing from $2.50 \AA$ to $2.78 \AA$ with $\mathrm{Au}$-Te equal to $2.45 \AA$.

A brief description of the energy levels and partial charge density distributions of the orbitals of AuCdSe and AuCdTe are shown in Figure 2 and Table 2, respectively. From the 


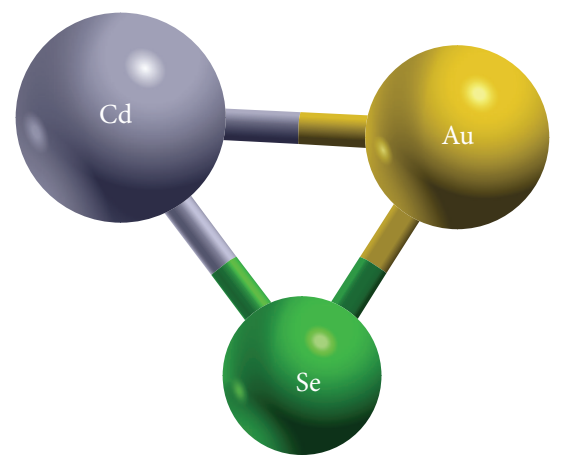

(a)

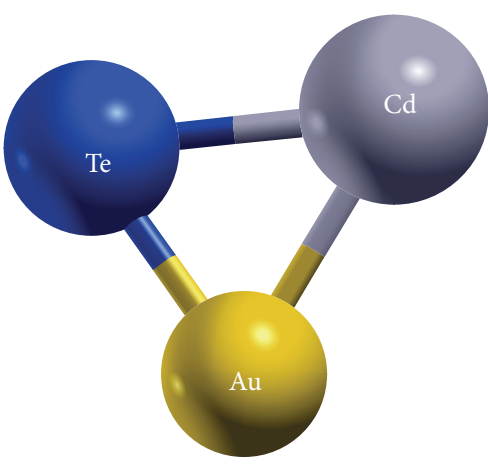

(b)

Figure 1: Minimum energy geometries of AuCdSe (a) and AuCdTe (b) trimers.

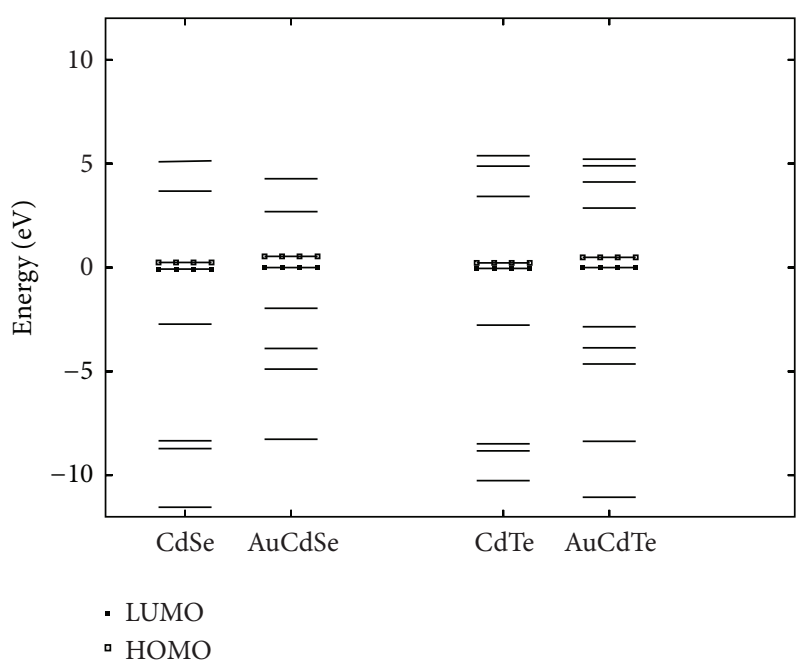

FIgure 2: Energy levels of AuCdSe and AuCdTe trimers as well as CdSe and CdTe dimers. The dark square represents the LUMO level while the HOMO levels are represented by white square, and the Fermi level is shifted to the zero of energy.

TABle 2: s, p, and d partial charges density within $\mathrm{Au}, \mathrm{Cd}, \mathrm{Se}$, and Te spheres calculated for some occupied and unoccupied orbitals in AuCdSe and AuCdTe clusters.

\begin{tabular}{ccccc}
\hline Orbital & Atom & $\mathrm{s}$ & $\mathrm{p}$ & $\mathrm{d}$ \\
\hline \multirow{4}{*}{ HOMO } & $\mathrm{Cd}$ & 0.11 & 0.04 & 0.01 \\
& $\mathrm{Se}$ & 0.00 & 0.14 & 0.00 \\
& $\mathrm{Au}$ & 0.00 & 0.01 & 0.06 \\
& $\mathrm{Cd}$ & 0.14 & 0.06 & 0.01 \\
& $\mathrm{Te}$ & 0.00 & 0.12 & 0.00 \\
& $\mathrm{Au}$ & 0.00 & 0.01 & 0.05 \\
\hline \multirow{4}{*}{ LUMO } & $\mathrm{Cd}$ & 0.03 & 0.00 & 0.00 \\
& $\mathrm{Se}$ & 0.00 & 0.12 & 0.00 \\
& $\mathrm{Au}$ & 0.10 & 0.05 & 0.03 \\
& $\mathrm{Cd}$ & 0.03 & 0.00 & 0.00 \\
& $\mathrm{Te}$ & 0.00 & 0.11 & 0.00 \\
& $\mathrm{Au}$ & 0.12 & 0.07 & 0.03 \\
\hline
\end{tabular}

energy level diagram it is seen that Fermi level of AuCdSe and AuCdTe clusters coincides with their respective $\mathrm{HOMO}$ levels. The LUMO level of CdSe and CdTe with the addition of $\mathrm{Au}$ atom moves up as compared to the position of the LUMO level of bare CdSe and CdTe dimers.

The evolution of the electronic structure of the cluster can be understood by studying the partial charge density of the HOMO and LUMO levels. Table 2 describes the s, p, and $\mathrm{d}$ partial charges density within the $\mathrm{Au}, \mathrm{Cd}, \mathrm{Se}$, and Te spheres calculated for some occupied and unoccupied orbitals in $\mathrm{AuCdSe}$ and AuCdTe trimers. The radius of the sphere of $\mathrm{Cd}, \mathrm{Se}, \mathrm{Au}$, and Te is considered to be $1.27 \AA, 1.05 \AA$, $1.21 \AA$, and $1.23 \AA$, respectively. The partial charges density calculated within the spheres of each atom, given in the table, denotes the atomic contribution to some of the energy levels around the Fermi level of AuCdSe and AuCdTe clusters. Figure 3 shows HOMO and LUMO partial charge density distribution of AuCdSe/Te clusters. It shows LUMO are localized on Se, $\mathrm{Te}$, and $\mathrm{Au}$, while almost all atoms contribute to the HOMO level.

To see easily the contribution of atoms on the HOMO and LUMO level of AuCdSe and AuCdTe trimers, Figure 4 well helps; in this figure the arrow points out which atom's levels contributed to the HOMO and LUMO level of AuCdSe and AuCdTe trimers.

The electronic transport properties of molecular devices can be obtained by studying the density of states (DOS) of the electrode-molecule-electrode system. The density of state along with the corresponding orbital energy levels of AuCdSe and AuCdTe clusters is shown in Figures 5(a) and 5(b), respectively. In both figures a high DOS at a specific energy level means states available for occupation but a zero DOS means that no state can be occupied at that energy level.

\subsubsection{Electronic Calculations of Planer Hexagonal Structure.} Figure 6(a) shows the geometrical structure of planer hexagonal $\mathrm{Cd}_{3} \mathrm{Se}_{3}$. Here it shows inward and outward relaxation of $\mathrm{Cd}$ and Se atoms, respectively. In this cluster, the $\mathrm{Cd}$ atoms form an equilateral triangle of side $3.09 \AA$ whereas the CdSe bond length is $2.44 \AA$. The binding energy per CdSe pair is calculated using (2) and is found to be $5.21 \mathrm{eV}$ which is 

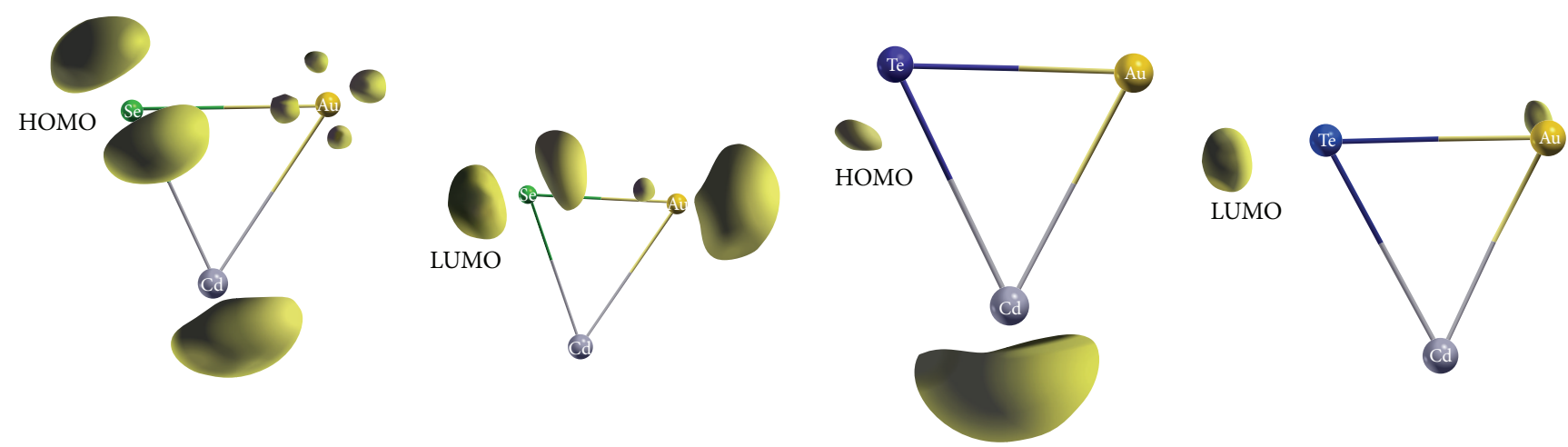

Figure 3: HOMO and LUMO of partial charge distribution of AuCdSe/Te trimers.

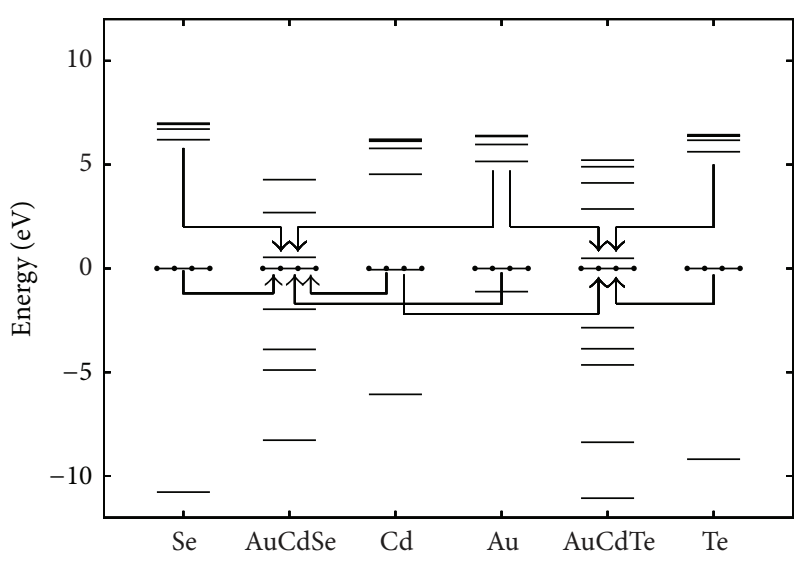

Figure 4: Energy levels of AuCdSe and AuCdTe. The energy levels of other components are depicted for comparison. Dotted lines represent Fermi energy levels for the respective systems, shown along $E=0$ axis.

approximately the same as the value reported by Zeweldi [17] and the HOMO-LUMO gap is calculated as $2.50 \mathrm{eV}$.

Figure 6(b) shows the geometrical structure of planer hexagonal $\mathrm{AuCd}_{2} \mathrm{Se}_{3}$. In this cluster, the geometry and electronic properties are slightly changed as compared to the structure of $\mathrm{Cd}_{3} \mathrm{Se}_{3}$ cluster including the bond lengths and angles. The average bond lengths of Cd-Se and Cd-Au are equal to $2.46 \AA$ and $2.90 \AA$, respectively. The bond lengths of $\mathrm{Au}-\mathrm{Se}$ and $\mathrm{Cd}-\mathrm{Cd}$ are equal to $2.38 \AA$ and $3.05 \AA$, respectively. The HOMO-LUMO gap is $2.65 \mathrm{eV}$ and the binding energy per atom is $2.87 \mathrm{eV}$.

Figure 6(c) shows the geometrical structure of planer hexagonal $\mathrm{AuCd}_{3} \mathrm{Se}_{2}$ cluster. In this cluster, the result indicated that there is a bond breakage between Cd-Cd atoms as compared to $\mathrm{Cd}_{3} \mathrm{Se}_{3}$ and $\mathrm{AuCd}_{2} \mathrm{Se}_{3}$ clusters. The average bond lengths of $\mathrm{Cd}-\mathrm{Se}, \mathrm{Cd}-\mathrm{Au}$, and $\mathrm{Cd}-\mathrm{Cd}$ are found to be $2.48 \AA, 2.56 \AA$, and $3.18 \AA$, respectively. The binding energy and HOMO-LUMO gap are calculated as $2.17 \mathrm{eV}$ and $1.45 \mathrm{eV}$, respectively.

Figure 6(d) shows the geometry of $\mathrm{Cd}_{3} \mathrm{Te}_{3}$ cluster which is similar to the structure of $\mathrm{Cd}_{3} \mathrm{Se}_{3}$ cluster. In this cluster the $\mathrm{Cd}$ atoms form an equilateral triangle of side $3.19 \AA$ whereas the $\mathrm{Cd}$-Te bond length is $2.62 \AA$. The binding energy per CdTe pair and the HOMO-LUMO gap are calculated as $4.61 \mathrm{eV}$ and $2.30 \mathrm{eV}$, respectively. The geometries obtained for the CdTe clusters are quite similar to the result reported by Bhattacharya and Kshirsagar [22].

Figure 6(e) shows the geometry of $\mathrm{AuCd}_{2} \mathrm{Te}_{3}$ cluster. In this cluster, the geometry and electronic properties are slightly changed as compared to the structure of $\mathrm{Cd}_{3} \mathrm{Te}_{3}$ cluster including the bond lengths and angles. The average bond lengths of $\mathrm{Cd}-\mathrm{Te}, \mathrm{Au}-\mathrm{Cd}$, and $\mathrm{Au}-\mathrm{Te}$ are equal to $2.63 \AA$, $2.96 \AA$, and $2.55 \AA$, respectively. The HOMO-LUMO gap and binding energy per atom are calculated as $2.63 \mathrm{eV}$ and $2.61 \mathrm{eV}$, respectively.

Figure 6(f) shows the geometry of $\mathrm{AuCd}_{3} \mathrm{Te}_{2}$ cluster. In this cluster, the result indicated that there is a bond breakage between Cd-Cd atoms as compared to $\mathrm{Cd}_{3} \mathrm{Te}_{3}$ and $\mathrm{AuCd}_{2} \mathrm{Te}_{3}$. The average bond lengths of $\mathrm{Cd}-\mathrm{Te}$ and $\mathrm{Cd}-\mathrm{Au}$ are found to be $2.65 \AA$ and $2.56 \AA$, respectively. The binding energy and HOMO-LUMO gap are calculated as $1.97 \mathrm{eV}$ and $1.47 \mathrm{eV}$, respectively.

Generally, replacing $\mathrm{Se} / \mathrm{Te}$ atom by $\mathrm{Au}$ atom in the structure of $\mathrm{Cd}_{3} \mathrm{Se}_{3} / \mathrm{Te}_{3}$, results indicate that the clusters have a narrow HOMO-LUMO gap. But $\mathrm{Cd}_{3} \mathrm{Se}_{3} / \mathrm{Te}_{3}$ and $\mathrm{AuCd}_{2} \mathrm{Se}_{3} / \mathrm{Te}_{3}$ clusters have greater value of binding energy and HOMO-LUMO gap, as compared to $\mathrm{AuCd}_{3} \mathrm{Se}_{2} / \mathrm{Te}_{2}$ trimer. Here $\mathrm{AuCd}_{3} \mathrm{Se}_{2} / \mathrm{Te}_{2}$ cluster is less stable than the other clusters.

3.1.4. Electronic Calculations of Au Atom Attached to Planer Hexagonal Structure. Figure 7 is the geometrical structure of planer hexagonal $\mathrm{Cd}_{3} \mathrm{Se}_{3} / \mathrm{Te}_{3}$ bonded to $\mathrm{Au}$ atom. In Figures $7(\mathrm{a})$ and 7 (c) the gold atom is found to be bonded with the $\mathrm{Cd}_{3} \mathrm{Se}_{3} / \mathrm{Te}_{3}$ clusters through the Se/Te atom, but in the case of Figures 7(b) and 7(d) the Au atom is bonded with the cluster through the $\mathrm{Cd}$ atom. Thus it shows different properties; for instance, the bond lengths of $\mathrm{Cd}-\mathrm{Se} / \mathrm{Te}$ as shown in Figure 7 vary due to the influence of gold atom. In addition to that Table 3 shows that a narrow HOMO-LUMO gap is observed if the $\mathrm{Au}$ atom approaches the $\mathrm{Cd}_{3} \mathrm{Se}_{3} / \mathrm{Te}_{3}$ cluster through the $\mathrm{Se} / \mathrm{Te}$ atom rather than through the $\mathrm{Cd}$ atom. But almost no effect is observed in binding energy.

3.1.5. Au Atom Bridging Two $\mathrm{Cd}_{3} \mathrm{Se}_{3} / \mathrm{Te}_{3}$ Clusters. Figure 8 shows the structure of two hexagonal planer $\mathrm{Cd}_{3} \mathrm{Se}_{3} / \mathrm{Te}_{3}$ 


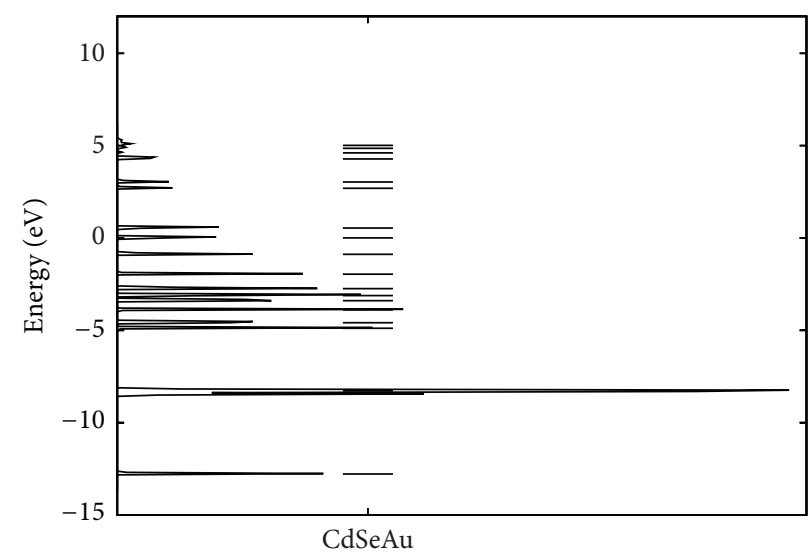

(a)

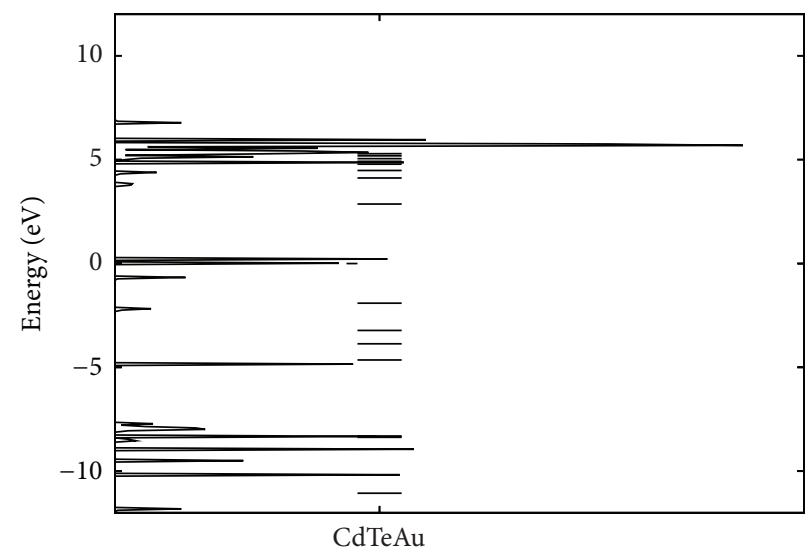

(b)

Figure 5: DOS and energy levels of CdSeAu (a) and CdTeAu (b) trimer. The discrete spectra are broadened by $0.02 \mathrm{eV}$ Gaussian smearing width.

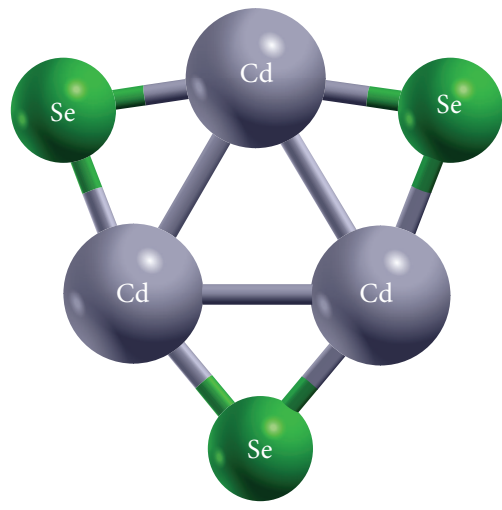

(a)

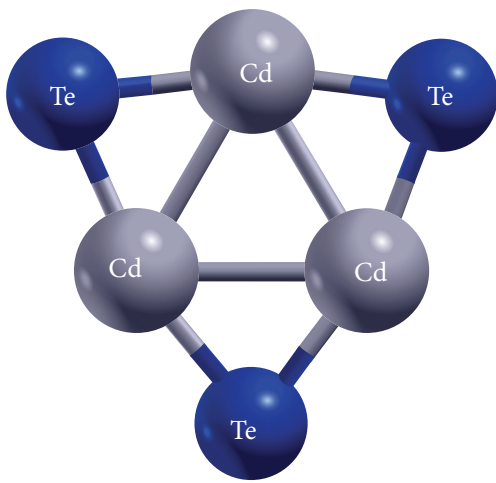

(d)

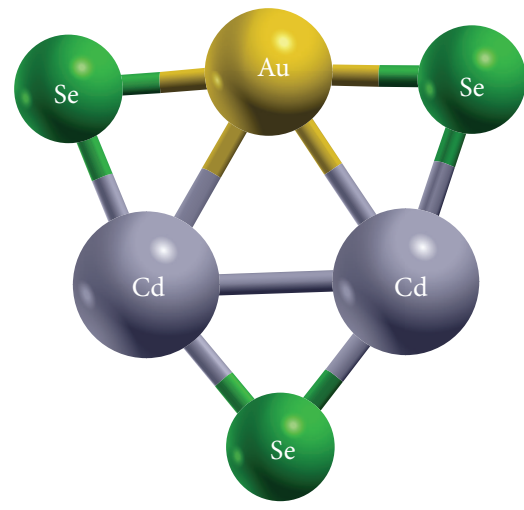

(b)

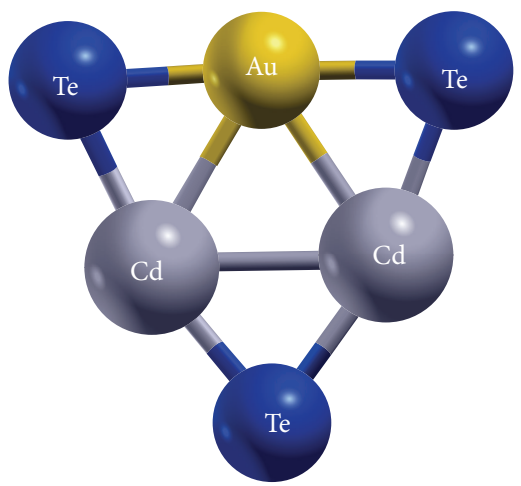

(e)

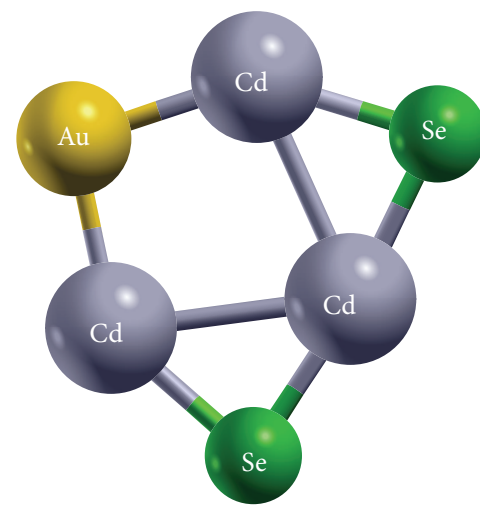

(c)

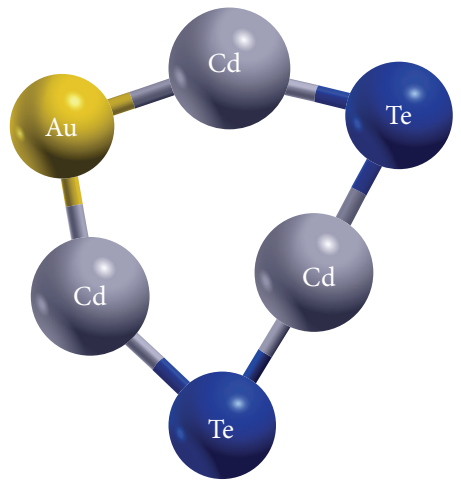

(f)

FIGURE 6: The minimum energy of planer hexagonal structure.

clusters bonded with single gold atom. In both clusters the influence of Au results in changing of properties; for instance, the bond length of $\mathrm{Cd}-\mathrm{Se} / \mathrm{Te}$ changes near the junction as compared to the edges. The binding energy and HOMOLUMO gap of the clusters are displayed in Table 4.

3.1.6. $\left(\mathrm{Au}_{13} \mathrm{Cd}_{3} \mathrm{Te}_{3}\right)_{2}$ and $\left(\mathrm{Au}_{13} \mathrm{Cd}_{3} \mathrm{Se}_{3}\right)_{2} \quad$ Nanodumbells. Before discussing the electronic properties of $\left(\mathrm{Au}_{13} \mathrm{Cd}_{3} \mathrm{Te}_{3}\right)_{2}$ and $\left(\mathrm{Au}_{13} \mathrm{Cd}_{3} \mathrm{Se}_{3}\right)_{2}$ nanodumbells, it is better to study electronic properties of bare $\mathrm{Au}_{13}, \mathrm{Cd}_{6} \mathrm{Te}_{6}$, and $\mathrm{Cd}_{6} \mathrm{Se}_{6}$ clusters.

Figure 9(a) shows the minimum energy structure of $A u_{13}$ cluster. The right face consists of four Au atoms forming a rhombus-type structure. These atoms serve as contact surface with the CdSe and CdTe nanowire. The average distance between the four atoms on the right face is $2.70 \AA$. The 


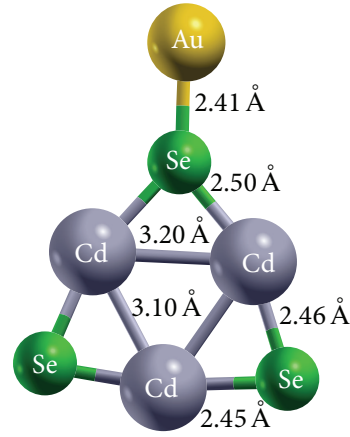

(a)

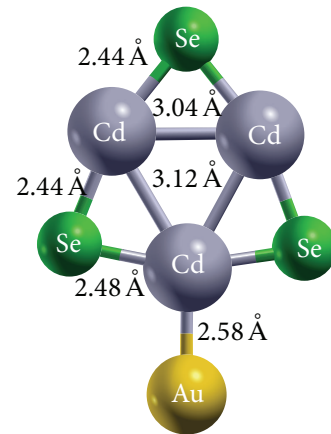

(b)

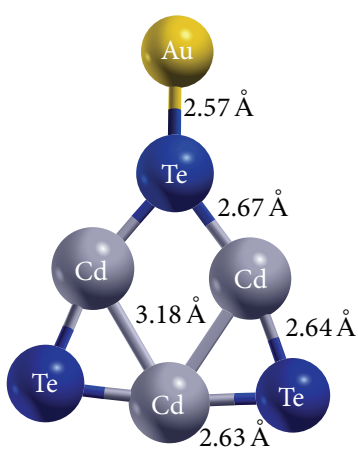

(c)

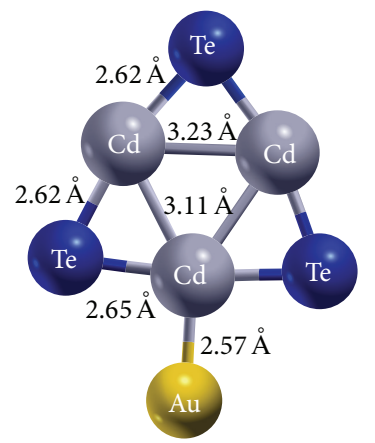

(d)

FIGURE 7: The minimum energy of Au atom attached to planer hexagonal structure $\mathrm{Cd}_{3} \mathrm{Se}_{3} / \mathrm{Te}_{3}$.

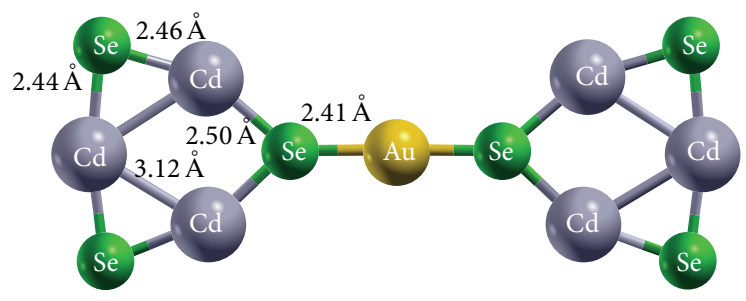

(a)

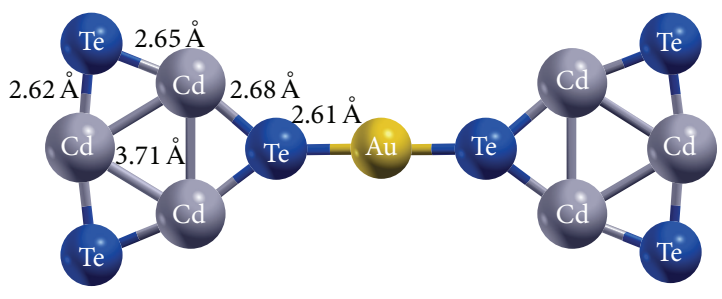

(b)

FIgURE 8: The minimum energy of Au atom bridging to two planers' hexagonal structure $\mathrm{Cd}_{3} \mathrm{Se}_{3} / \mathrm{Te}_{3}$.

TABLE 3: Electronic calculation of a planer $\mathrm{Cd}_{3} \mathrm{Se}_{3} / \mathrm{Te}_{3}$ cluster attached to Au atom.

\begin{tabular}{lcc}
\hline Figure 7 & $\mathrm{BE}(\mathrm{eV})$ & $\begin{array}{c}\text { HOMO-LUMO } \\
\text { gaps }(\mathrm{eV})\end{array}$ \\
\hline Figure 7(a) & 2.39 & 1.36 \\
Figure 7(b) & 2.39 & 2.93 \\
Figure 7(c) & 2.14 & 1.40 \\
Figure 7(d) & 2.15 & 2.63 \\
\hline
\end{tabular}

TABLE 4: Electronic calculation of two planer $\mathrm{Cd}_{3} \mathrm{Se}_{3} / \mathrm{Te}_{3}$ clusters' bridging by $\mathrm{Au}$ atom.

\begin{tabular}{lcc}
\hline Figure 8 & $\mathrm{BE}(\mathrm{eV})$ & $\begin{array}{c}\text { HOMO-LUMO } \\
\text { gaps }(\mathrm{eV})\end{array}$ \\
\hline Figure 8(a) & 2.58 & 0.67 \\
Figure 8(b) & 2.30 & 0.85 \\
\hline
\end{tabular}

binding energy per atom and the HOMO-LUMO gaps are $2.99 \mathrm{eV}$ and $0.80 \mathrm{eV}$, respectively, approximately the same as those presented in [17]. The average bond length of $\mathrm{Au}-\mathrm{Au}$ is found to be $2.69 \AA$ which is exceeded by $0.22 \AA$ from the $A u$ dimer.

Figure 9(b) shows the wurtzite geometry of $\mathrm{Cd}_{6} \mathrm{Te}_{6}$ having an average bond length of Cd-Te equal to $2.72 \AA$ (on the layers) and $2.87 \AA$ (between the layers). Binding energy (per CdTe pair) and HOMO-LUMO gap are calculated as $5.08 \mathrm{eV}$ and $1.86 \mathrm{eV}$, respectively.

Figure 9(c) shows the wurtzite geometry structure of $\mathrm{Cd}_{6} \mathrm{Se}_{6}$ having an average bond length of $\mathrm{Cd}$-Se equal to $2.54 \AA$ (on the layers) and $2.74 \AA$ (between the layers). Binding energy (per CdSe pair) and HOMO-LUMO gap are calculated as $5.68 \mathrm{eV}$ and $2.02 \mathrm{eV}$, respectively. A similar structure and binding energy are reported by Deglmann et al. [23].

The geometry of $\mathrm{Au}_{13}\left(\mathrm{Cd}_{3} \mathrm{Se}_{3}\right)_{2}$ is shown in Figure 10(a). The average bond length of $\mathrm{Cd}-\mathrm{Se}$ is $2.61 \AA$ at the center and $2.72 \AA$ near the junction. This is an indication of the compactness of the nanowire at the center compared with that at the junction.

The average bond lengths of $\mathrm{Cd}-\mathrm{Au}, \mathrm{Se}-\mathrm{Au}$, and $\mathrm{Au}-\mathrm{Au}$ are equal to $2.76 \AA, 2.54 \AA$, and $2.71 \AA$, respectively. The binding energy and HOMO-LUMO gap are found to be $3.26 \mathrm{eV}$ and $0.55 \mathrm{eV}$, respectively.

The geometry of $\left(\mathrm{Au}_{13} \mathrm{Cd}_{3} \mathrm{Te}_{3}\right)_{2}$ is shown in Figure $10(\mathrm{~b})$. The average Cd-Te bond length is found to be $2.85 \AA$ at the center and $2.93 \AA$ near the junction. This is similar to the $\left(\mathrm{Au}_{13} \mathrm{Cd}_{3} \mathrm{Se}_{3}\right)_{2}$ cluster discussed above, compactness of the nanowire at the center compared with that at the junction. The average bond lengths of $\mathrm{Cd}-\mathrm{Au}$ and $\mathrm{Te}-\mathrm{Au}$ are equal to $2.64 \AA$ and $2.66 \AA$, respectively. The binding energy, calculated using (2), and the HOMO-LUMO gap are found to be $3.18 \mathrm{eV}$ and $0.31 \mathrm{eV}$, respectively.

Generally, when we see the properties different in these two nanodumbells, $\left(\mathrm{Au}_{13} \mathrm{Cd}_{3} \mathrm{Se}_{3}\right)_{2}$, have greater binding and HOMO-LUMO gap energies, $\left(\mathrm{Au}_{13} \mathrm{Cd}_{3} \mathrm{Te}_{3}\right)_{2}$ cluster is less stable than $\left(\mathrm{Au}_{13} \mathrm{Cd}_{3} \mathrm{Se}_{3}\right)_{2}$ cluster.

Figure 11 displays LDOS of the $\mathrm{Au}_{13}\left(\mathrm{Cd}_{3} \mathrm{Se}_{3}\right)_{2}$ nanodumbell. Panels (I), (II), and (III) display the LDOS calculated in the corresponding different regions as defined in the geometry of nanodumbell, which is similar result to the value 


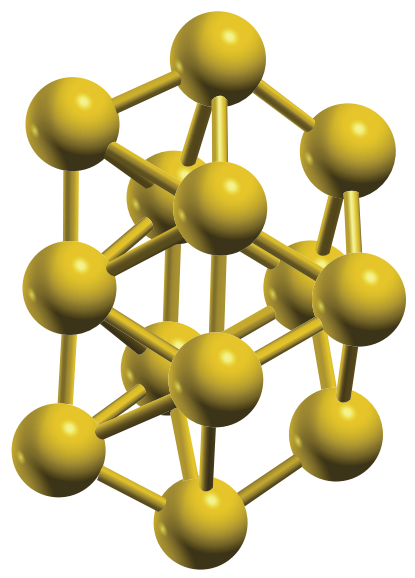

(a)

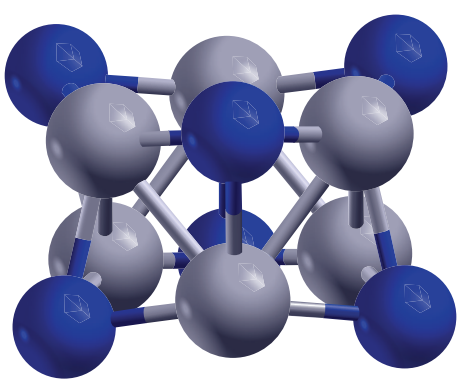

(b)

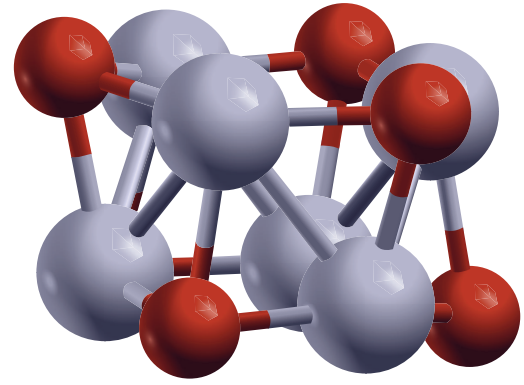

(c)

FIGURE 9: Geometrical structure of $\mathrm{Au}_{13}$ (a), $\mathrm{Cd}_{6} \mathrm{Se}_{6}$ (b), and $\mathrm{Cd}_{6} \mathrm{Te}_{6}$ (c) clusters.

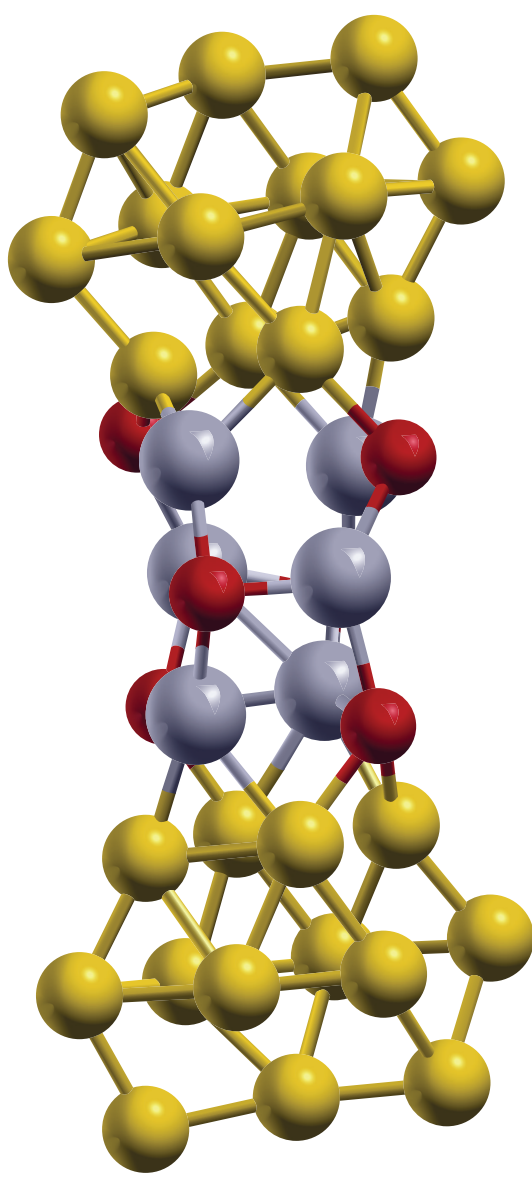

(a)

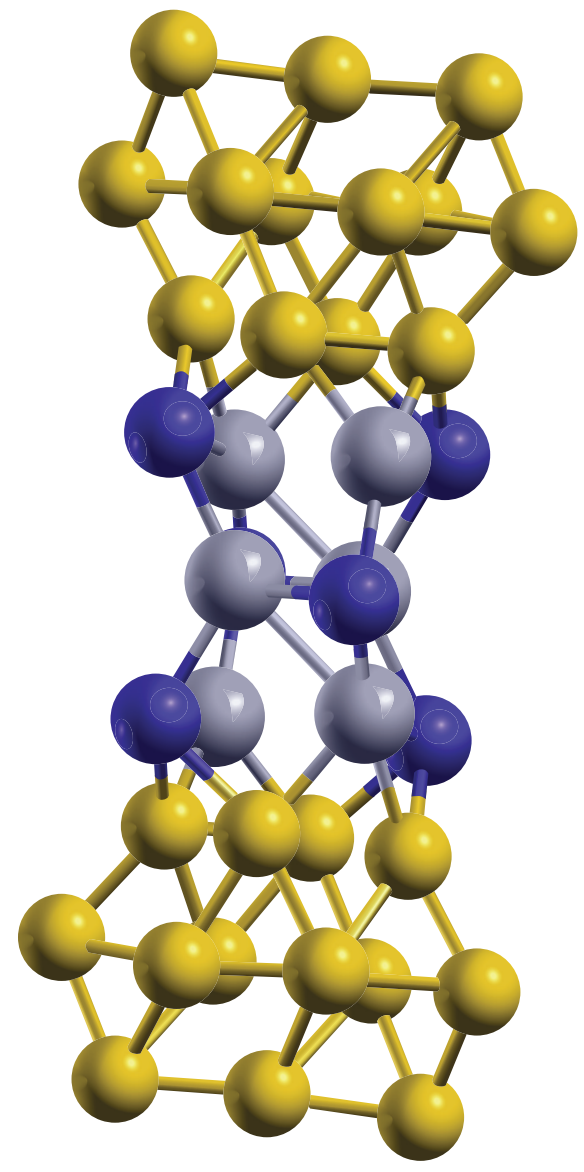

(b)

FIGURE 10: Geometry of $\left(\mathrm{Au}_{13} \mathrm{Cd}_{3} \mathrm{Se}_{3}\right)_{2}$ (a) and $\left(\mathrm{Au}_{13} \mathrm{Cd}_{3} \mathrm{Te}_{3}\right)_{2}$ (b) nanodumbells.

calculated by Ghebriel and Kshirsagar [24]. Panel (TDOS) shows the whole DOS of $\mathrm{Au}_{13}\left(\mathrm{Cd}_{3} \mathrm{Se}_{3}\right)_{2}$ nanodumbell. Panel (I), showing the LDOS of the four Au atoms at the junction, has similar characteristics to the DOS in panel (TDOS). Panel (II) is the LDOS of CdSe atoms in contact with the Au lead.
In panels (I and II), almost there is no significant difference in LDOS plots between them. This is an indication that the $\mathrm{CdSe}$ atoms in contact with the $\mathrm{Au}$ atoms are metalized. Panel (III) displays the LDOS of CdSe atoms at the center of the nanowire. There are a few states in region (III) around the 

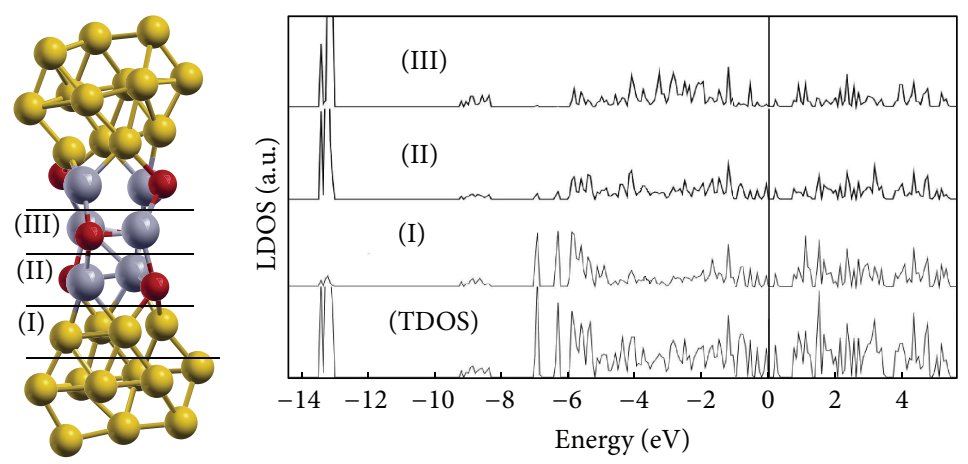

FIGURE 11: LDOS for $\mathrm{Au}_{13}\left(\mathrm{Cd}_{3} \mathrm{Se}_{3}\right)_{2}$ nanodumbell; panel (TDOS) displays the total DOS and panels (I-III) display LDOS calculated in different regions. The discrete spectra are broadened by $0.02 \mathrm{eV}$ Gaussian smearing width. The vertical line at $E=0$ denoted Fermi energy levels.

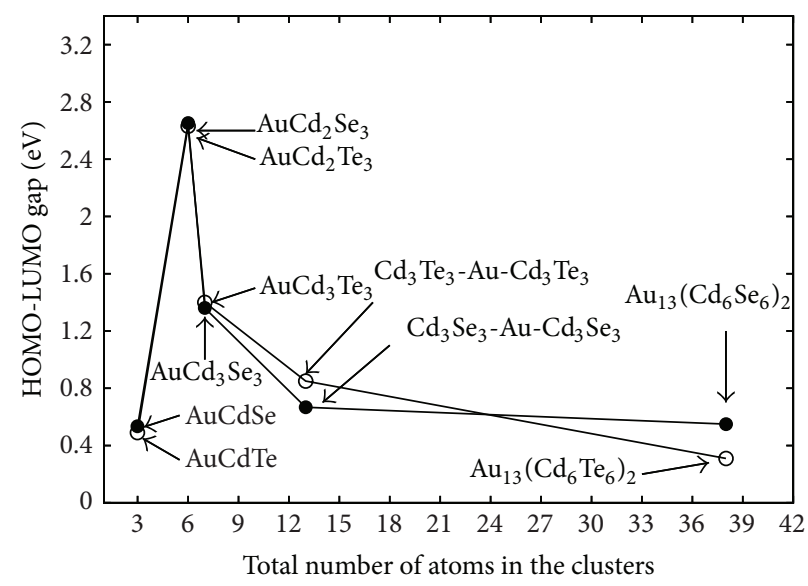

(a)

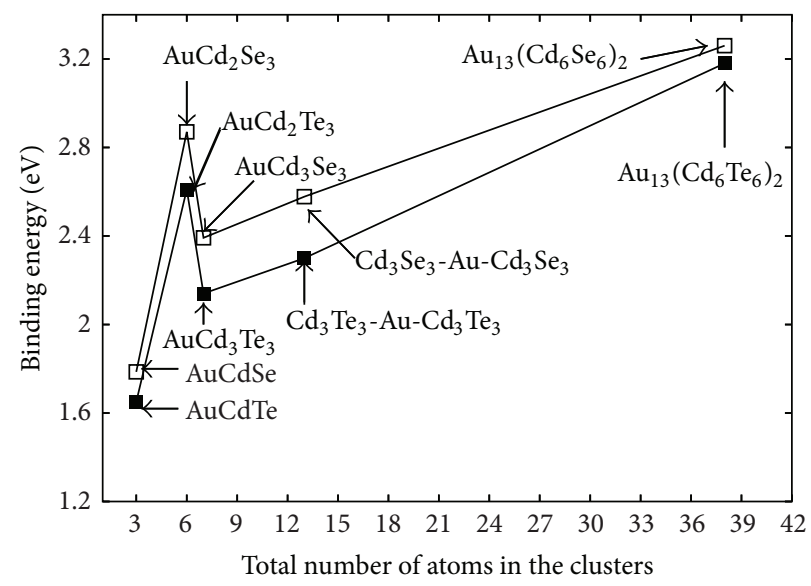

(b)

FIgURE 12: HOMO-LUMO gap and binding energy of $\mathrm{Au}_{l} \mathrm{Cd}_{m} \mathrm{Se}_{n}$ and $\mathrm{Au}_{l} \mathrm{Cd}_{m} \mathrm{Te}_{n}$ clusters.

Fermi level as compared regions (I and II). Hence, we say that the $\mathrm{Cd}_{6} \mathrm{Se}_{6}$ nanowire shows metallic property when it is sandwiched between $\mathrm{Au}_{13}$ nanoelectrodes.

\subsection{The Effect of Size on the Properties of Clusters. Figure 12(a)} shows the HOMO-LUMO gap versus numbers of atoms in the clusters $\mathrm{Au}_{l} \mathrm{Cd}_{m} \mathrm{Se}_{n}$ and $\mathrm{Au}_{l} \mathrm{Cd}_{m} \mathrm{Te}_{n}$. The figure clearly shows that the HOMO-LUMO gap decreases with increasing number of atoms in both clusters except in the trimers. In other words, the HOMO-LUMO gap is inversely proportional to the size of the clusters.

Figure 12(b) shows the binding energy versus numbers of atoms in the clusters $\mathrm{Au}_{l} \mathrm{Cd}_{m} \mathrm{Se}_{n}$ and $\mathrm{Au}_{l} \mathrm{Cd}_{m} \mathrm{Te}_{n}$. The figure generally points out that the binding energy increases with increasing cluster size, with the exception of $\mathrm{AuCd}_{2} \mathrm{Se}_{3} / \mathrm{Te}_{3}$ clusters where the binding energy is large as compared to neighboring clusters. Clusters of certain sizes often have special properties such as higher stability or larger gap when compared to other clusters. The correlation between gap size and stability can be justified by simple tight binding descriptions in which the highest occupied molecular orbital(HOMO-) lowest unoccupied molecular orbital (LUMO) gap increased the orbital overlap [25]. We found that clusters with 6 atoms have substantially higher HOMO-LUMO gaps, and they also exhibit local maxima in the binding energies. Therefore, clusters $\mathrm{AuCd}_{2} \mathrm{Se}_{3}$ and $\mathrm{AuCd}_{2} \mathrm{Te}_{3}$ are more stable than others.

\section{Summary and Conclusion}

In this paper the electronic structures of cadmium selenide and cadmium telluride attached to gold atoms have been presented. The electronic structures were simulated by using VASP, a simulation package which is based on the principle of density functional theory (DFT).

The simulations of cadmium selenide and cadmium telluride clusters attached to gold atoms are performed to study the size effect of electronic structures of the clusters, particularly bandgap (HOMO-LUMO gap), and binding energy and the partial charge density distributions of the HOMO and LUMO orbitals were studied. Two respective structures are optimized for the $\mathrm{CdSe} / \mathrm{Te}$ dimers attached to $\mathrm{Au}$ atom and we have found that the linear structures are at higher energy than the triangular geometries. 
Replacing $\mathrm{Cd}$ atom by $\mathrm{Au}$ atom in the structure of $\mathrm{Cd}_{3} \mathrm{Se}_{3} / \mathrm{Te}_{3}$ clusters; results indicated that relatively more stable than replacing Se/Te atoms. In this work we have also found a relatively higher stability when two planer $\mathrm{Cd}_{3} \mathrm{Se}_{3} / \mathrm{Te}_{3}$ clusters are connected by bridging $\mathrm{Au}$ atom rather than bridging with $\mathrm{Ag}$ atom. The LDOS graph of $\mathrm{Au}_{13}\left(\mathrm{Cd}_{3} \mathrm{Se}_{3}\right)_{2}$ indicated that the $\mathrm{Cd}_{6} \mathrm{Se}_{6}$ nanowire shows metallic property when it is sandwiched between $\mathrm{Au}_{13}$ nanoelectrodes.

The graph of HOMO-LUMO gap versus number of atoms in the cluster shows an increase in gap with decreasing cluster size. This dependence of HOMO-LUMO gap on the size of the cluster is in agreement with the theory of nanostructures. From the graph of binding energy versus number of cluster atoms, it is found that the binding energy increases with increasing cluster size, with the exception of $\mathrm{AuCd}_{2} \mathrm{Se}_{3} / \mathrm{Te}_{3}$ clusters where the binding energy is large as compared to neighboring clusters. In this work clusters with 6 atoms have substantially higher HOMO-LUMO gaps, and they also exhibit local maxima in the binding energies. Therefore, $\mathrm{AuCd}_{2} \mathrm{Se}_{3}$ and $\mathrm{AuCd}_{2} \mathrm{Te}_{3}$ are more stable in comparison with their neighbors. These special clusters $\left(\mathrm{AuCd}_{2} \mathrm{Se}_{3}\right.$ and $\mathrm{AuCd}_{2} \mathrm{Te}_{3}$ ) have contributed to the unique attributes of the novel transistors such as single electron transistor (SET). Hence as a further work we recommend a detailed study of the band structure and electron transport in SET.

\section{Conflict of Interests}

The authors declare that there is no conflict of interests regarding the publication of this paper.

\section{Acknowledgments}

This work was supported by the Ethiopian Ministry of Education (MoE). First and foremost, B. Shimeles would like to thank the almighty God. Finally, B. Shimeles wish to express his profound gratitude to his father for laying the groundwork in his life to accomplish this.

\section{References}

[1] The Royal Society and The Royal Academy of Engineering, Nanotechnology and Nanoscience, 2004.

[2] NASA Ames Research Center, Nanotechnology Opportunities and Challenges, 2005.

[3] M. M. S. El-Tonsy, "Nanotechnology and nanomedicine applications in parasitic diseases," Parasitologists United Journal, vol. 3, no. 1, pp. 19-26, 2010.

[4] P. Jena and S. N. Behera, Clusters and Nanostructured Materials, Nova Science Publishers, 1996.

[5] C. Q. Sun, "Size dependence of nanostructures: impact of bond order deficiency," Progress in Solid State Chemistry, vol. 35, no. 1, pp. 1-159, 2007.

[6] C. P. Poole Jr. and F. J. Owens, Introduction to Nanotechnology, Wiley, 2003.

[7] J. G. Vilhena, S. Botti, and M. A. L. Marques, "Excitonic effects in the optical properties of CdSe nanowires," Applied Physics Letters, vol. 96, no. 12, Article ID 123106, 2010.
[8] P. T. Barton, "Formation of Metal-semiconductor axial nanowire heterostructures through controlled silicidation," 2009.

[9] G. Kresse and J. Furthmüller, VASP the GUIDE, 2005.

[10] Y. Horie, Shock Wave Science and Technology Reference Library, 2009.

[11] D. Vanderbilt, "Soft self-consistent pseudopotentials in a generalized eigenvalue formalism," Physical Review B, vol. 41, no. 11, pp. 7892-7895, 1990.

[12] R. Armiento, Themany-Electron Energy in Density Functional Theory, 2005.

[13] D. M. Ceperley and B. J. Alder, "Ground state of the electron gas by a stochastic method," Physical Review Letters, vol. 45, no. 7, pp. 566-569, 1980.

[14] T. Körzdörfer and S. Kümmel, "Single-particle and quasiparticle interpretation of Kohn-Sham and generalized Kohn-Sham eigenvalues for hybrid functional," Physical Review B, vol. 82, no. 15, Article ID 155206, 2010.

[15] C. Kittel, Introduction to Solid State Physics, Wiley, Hoboken, NJ, USA, 2005.

[16] G. A. Mansoori, Principles of Nanotechnology: Molecular-Based Study of Condensed Matter in Small System, World Scientific Publishers, 2006.

[17] H. W. Zeweldi, Density functional study of electronic and structural properties of different gold cluster-complexes [Ph.D. thesis], University of Pune, Pune, India, 2008.

[18] C. Majumder and S. K. Kulshreshtha, "Structural and electronic properties of $\mathrm{Au}_{n}(n=2-10)$ clusters and their interactions with single $S$ atoms: Ab initio molecular dynamics simulations," Physical Review B, vol. 73, Article ID 155427, 2006.

[19] R. David, CRC Handbook of Chemistry and Physics, CRC Press, 2008.

[20] H. Sellers, A. Ulman, Y. Shnidman, and J. E. Eilers, "Structure and binding of alkanethiolates on gold and silver surfaces: implications for self-assembled monolayers," The Journal of the American Chemical Society, vol. 115, no. 21, pp. 9389-9401, 1993.

[21] E. Sanville, A. Burnin, and J. J. BelBruno, "Experimental and computational study of small $(n=1-16)$ stoichiometric zinc and cadmium chalcogenide clusters," The Journal of Physical Chemistry A, vol. 110, no. 7, pp. 2378-2386, 2006.

[22] S. K. Bhattacharya and A. Kshirsagar, " $A b$ initio calculations of structural and electronic properties of CdTe clusters," Physical Review B, vol. 75, no. 3, Article ID 035402, 10 pages, 2008.

[23] P. Deglmann, R. Ahlrichs, and K. Tsereteli, “Theoretical studies of ligand-free cadmium selenide and related semiconductor clusters," Journal of Chemical Physics, vol. 116, no. 4, pp. 15851597, 2002.

[24] H. W. Ghebriel and A. Kshirsagar, "Electronic structure of Cdse nanowires terminated with gold electrodes," Momona Ethiopian Journal of Science, vol. 3, no. 1, pp. 5-19, 2011.

[25] M. C. Troparevsky and J. R. Chelikowsky, "Structural and electronic properties of CdS and CdSe clusters," Journal of Chemical Physics, vol. 114, no. 2, pp. 943-949, 2001. 

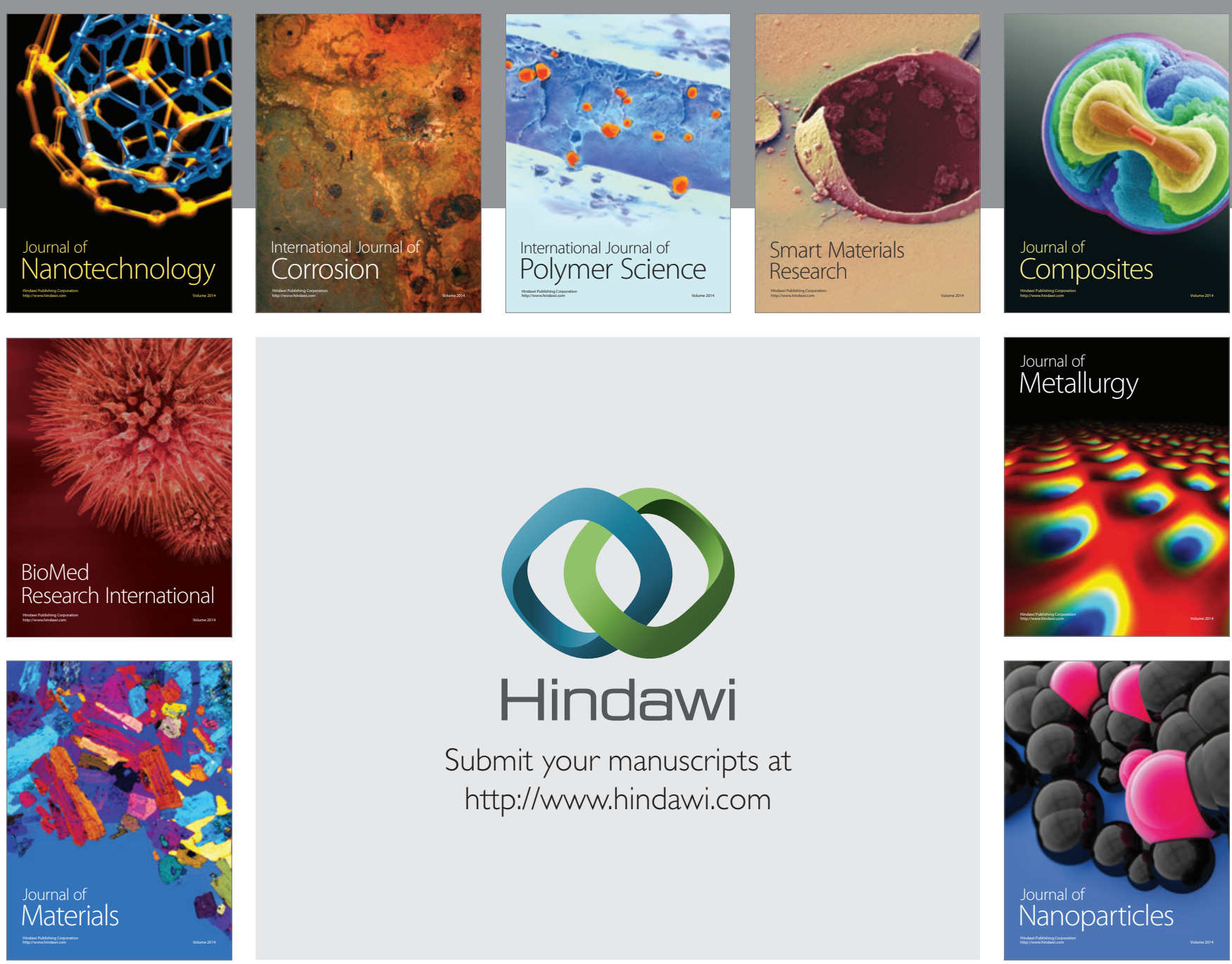

Submit your manuscripts at http://www.hindawi.com
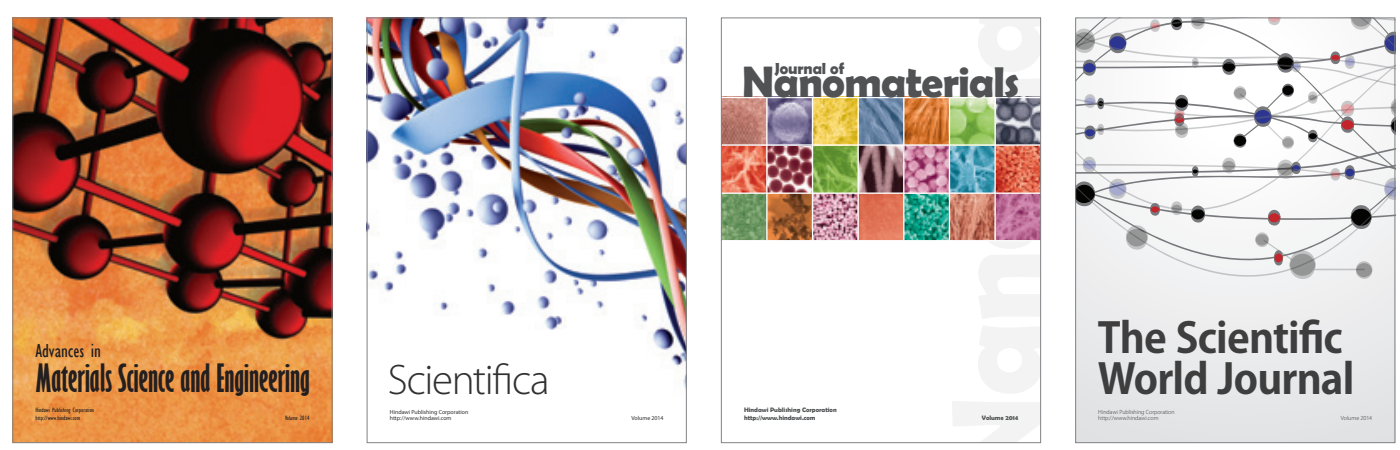

\section{The Scientific World Journal}
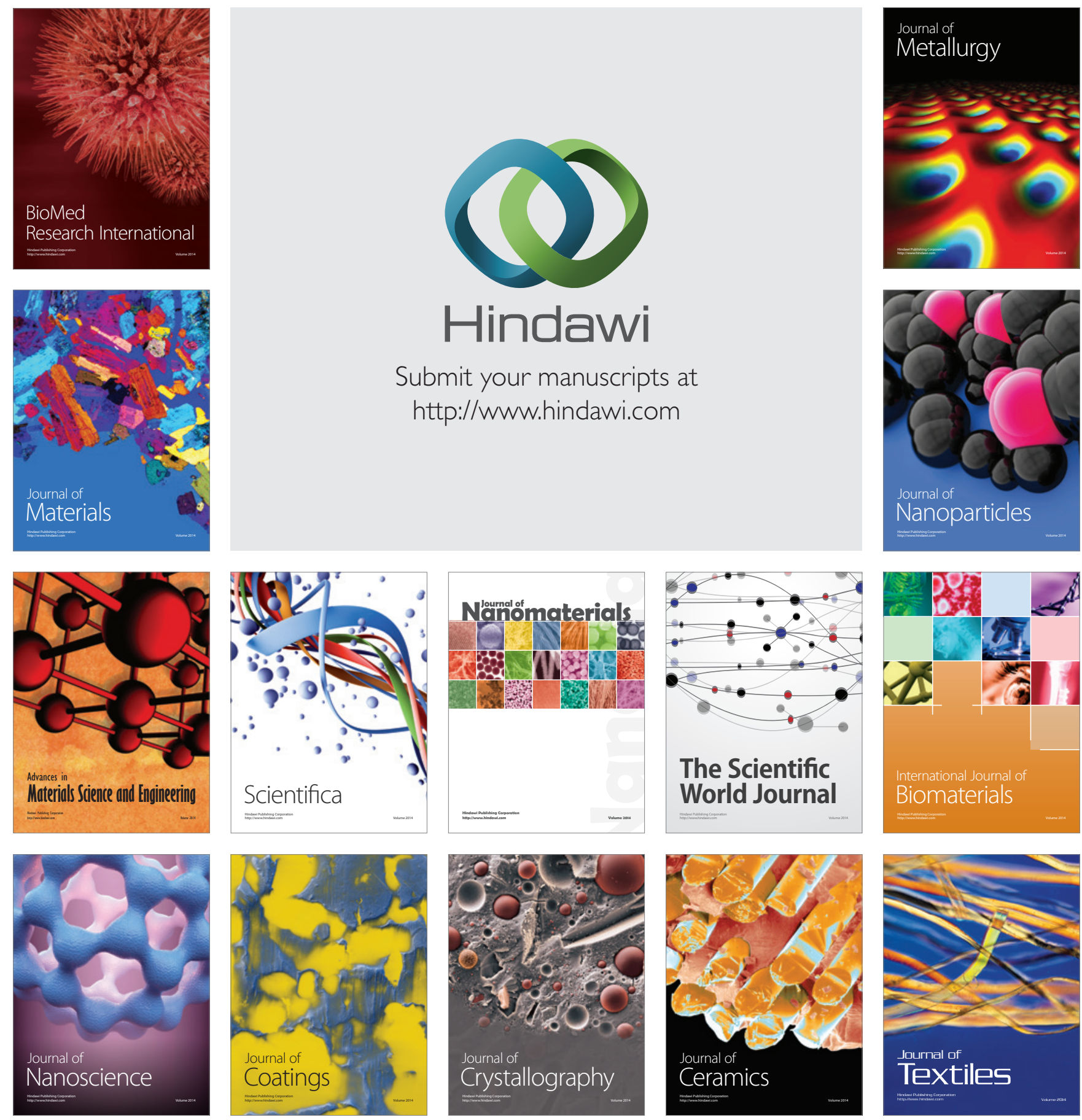